\section{Pups and groundhogs}

\author{
Alan Hendry \\ Dictionary of Ceramic Science and \\ Engineering. \\ By Loran S. O'Bannon. \\ Plenum: 1984. Pp.303. \$45, £42.75.
}

"CERAMIC science and engineering is one of the oldest yet one of the newest, fastest growing ... of man's endeavours". Dr O'Bannon begins his preface to the Dictionary of Ceramic Science and Engineering with this comment, and therein lies the problem for the etymologist. Ancient origins and the speed with which ceramic science is expanding and incorporating terms from associated disciplines make it impossible to provide a definitive compilation.

Dr O'Bannon recognizes these problems, and based on his wide experience of the subject he has made a brave attempt at providing a reference book to suit several tastes. In so doing, however, he has fallen between several stools. The Dictionary is not detailed enough for the professional ceramist and yet is too detailed for nonceramists. Extensive sections are devoted to rare earth compounds but the data are of limited value. The information on applications is useful but the representation used in giving chemical data is misleading; for example, the "silicon carbide composite" $\mathrm{SiC} . \mathrm{Si}_{3} \mathrm{~N}_{4}$, molecular weight 180.28 , would be taken to imply a chemical compound of one molecule $\mathrm{SiC}$ and one molecule $\mathrm{Si}_{3} \mathrm{~N}_{4}$, but which does not in fact exist.

The book is dedicated to promoting the standardization of terms in ceramic science and engineering and any attempt in this direction, particularly in a trans-Atlantic context, is to be commended. The bibliography provides a useful source from which to follow up the information contained in the text. Dr Johnson remarked that "making dictionaries is dull work", but omitted to mention that when made they are useful; in both senses this volume is no exception. Readers will no doubt find definitions with which they disagree, but sufficient reason for buying the book is that there is no better alternative available.

Alan Hendry is Joint Director of the Wolfson Research Group for High Strength Materials and a Lecturer in Metallurgy at the University of Newcastle upon Tyne.

\section{Business in science}

\section{W.F. Clocksin}

\section{Dictionary of Computers, Data}

Processing and Telecommunications. By Jerry M. Rosenberg.

Wiley: 1984. Pp. 614. Hbk f29.95,

\$29.95; $p b k$ f14.95, \$14.95.

Dictionary of Computing.

Oxford University Press: 1984. Pp. 393. f15, \$25.

AT ONE and the same time computing is a thriving academic subject, a growth industry as well as a new hobby for many. Computer science, in which the fundamental principles of computation are studied, is a subject area of prime interest in universities and research centres. There has been explosive growth in the applications of computing to business problems, including commercial data processing, telecommunications and the technology of information handling. Such a rapidly expanding and diverse field calls for up-to-date reference books which bring together the principles with the practice.

The large Dictionary of Computers, Data Processing and Telecommunications is only partly a success. Containing more than 10,000 entries, it is directed mainly towards the commercial applications. Many entries are in fact extracts from other dictionaries and technical standards documents, while others are a mixture of marketing slogans and singular neologisms; I am uncertain whether it is justifiable to confer respectability on such terms by documenting them in this way. Typographical errors are in evidence, and there are some spectacular blunders, such as equatwith the holes punched in paper cards. Together with other examples, this rein-

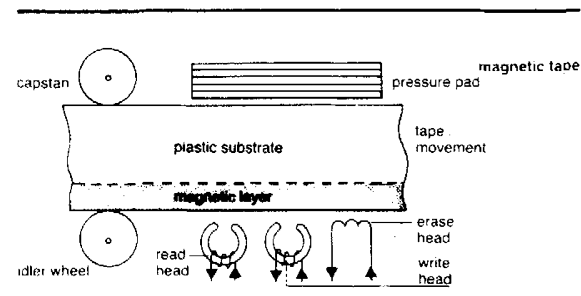

Piclure "magnetic tape" and "read head"' (Reproduced from the Cambridge Illustrated Thesaurus of Computer Science, by Arthur Godman, published by Cambridge University Press price $£ 4.95$.)

forces my impression that the author does not know the territory well.

I prefer the Dictionary of Computing. With about 3,800 well-chosen entries it is much smaller than the previous volume, but it is superbly edited, with instructive entries and intelligent cross-referencing. Terms in computer science are strongly represented, but the commercial world of data processing is not ignored. Unlike Rosenberg's book, some entries include useful drawings and tables. This is a valuable and reliable reference for the student as well as the computing professional.

W.F. Clocksin is an Assistant Director of Research at the Computer Laboratory, University of Cambridge. ing the "holes" in semiconductor materials
In terms of computer graphics

\section{Julian Gallop}

Dictionary of Computer Graphics.

By John Vince.

Frances Pinter, London/Knowledge

Industries Publishing, White Plains, New

York: 1984. Pp. 132. £15, \$34.95.

ONE might expect a dictionary on a technical topic to contain rather formal definitions of a number of terms. In Dictionary of Computer Graphics John Vince does indeed follow the structure of alphabetically ordered entries, but he has chosen to unite them in a descriptive manner with generous use of figures. This gives a good introduction to the chosen topics, even if a few entries are rather longer than is usual in a dictionary. There is a slight danger in the simplicity that this encourages; for example, a statement made under "hidden-line removal" about a regular polyhedron can in fact be made with more generality about a convex polyhedron and would give more insight into the reasons for the statement. However the overall style does encourage the reader to explore the book.

My reservations are mainly about the organization of the Dictionary and the selection of terms for inclusion. There are cases where the specific can be consulted, but not the general; for instance one can look up Bézier curves (and patches) but not discover that B-spline is an alternative technique described in the book - if curvefitting were an entry it could refer to both. In a field where terminology has not stabilized, and several terms are used for similar concepts, a dictionary has a duty to record them, because the reader will come across those terms elsewhere; thus picture plane is defined in the context of two-dimensional projections of three-dimensional scenes, but not projection plane or view plane, which are used in a well-regarded textbook.

Some terms used internally are not defined, while others are defined as a group; thus PAL, the method of encoding television signals used in Britain is described but cannot be looked up because it appears under NTSC which is the American system. The person learning to program some computer graphics applications will not find any mention of such commonly accepted terms as segment, viewport or pick. Possibly the book is not intended for such people.

The Dictionary could, then, have been more thorough and balanced. But it is attractively written and presented, and conveys an infectious enthusiasm for the subject.

Julian Gallop is a Senior Scientific Officer in the Computer Graphics Section at the Rutherford Appleton Laboratory, Chilton, Oxfordshire. 